\title{
The simulation and optimization of a multi-basin water resource scheme in Cyprus
}

\author{
J. K. WHITE \& C. A. CHRISTOdOULOU
}

Dr R. M. Jarrold, Hunting Technical Services, CICA. Santiago

Work in Chile on a potential development of four interconnected river basins has led to the development of a planning model which is similar in many respects to that used for the Cyprus projects.

53. I would like to ask some questions which concern the underlying assumptions of the model. The Authors state $(\$ 29)$ that 'annual production . . . is reduced on account of any water deficits found in the water balance.' How was this reduction evaluated?

54. I think this problem is critical to the outcome of the whole analysis. Different crops, with their own specific water requirements, are affected differently by deficits in any given month, and a deficit of a given magnitude has a different effect on the yield (i.e. benefit) of a crop depending on the month in which it occurs. In other words, to calculate the reduction in yield for a range of crops within a given cropping pattern, one requires yield response/water demand curves for each crop, in each month, in each climatic/soil zone.

55. Can the Authors give the data they used for this purpose? Precise information is not available in Chile.

56. When deficits occur farmers must decide which crop to sacrifice. If they were to act rationally, they would stop watering the crop which provides the lowest marginal return to water, at the time the deficit occurs. This raises a problem because, following from the yield/water response relationship, marginal returns to water vary from month to month and hence the order of sacrifice varies throughout the growing season. How did the Authors deal with this problem? In Chile a constant order of sacrifice has been adopted (for each climatic zone) based on marginal returns to water in the most critical month in terms of water deficit. This is clearly an oversimplification and yet to vary the order of sacrifice from one month to another complicates the model and increases running costs.

57. Traditionally, hydrological security is defined as the number of years out of 100 when supply equals or exceeds demand. With this definition, it is customary to select a given security level for design purposes and commonly the figure adopted is $85 \%$. In Chile it has been concluded that the optimum security level should be determined for each potential project and it has been found that this varies from project to project. Furthermore, economic security, defined as the probability of achieving full benefits, has been found to be approximately $10 \%$ higher than the estimated hydrological security, i.e. with $85 \%$ security of supply, farmers obtain approximately $95 \%$ of potential

Paper published: Proc. Instn Civ. Engrs, Part 2, 1979, 67, Mar., 111-132. 


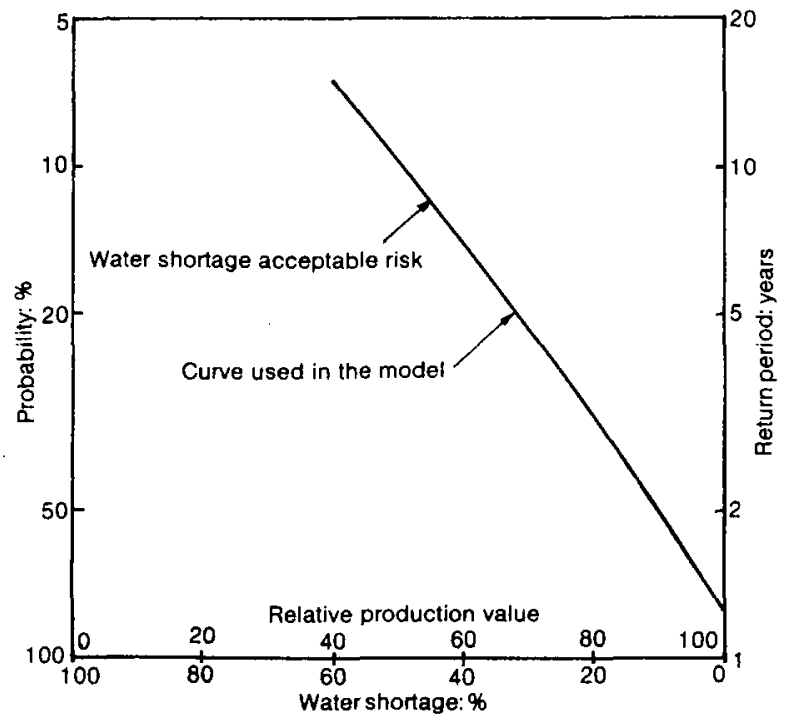

Fig. 6. Water shortage and crop failure frequency analysis for bananas

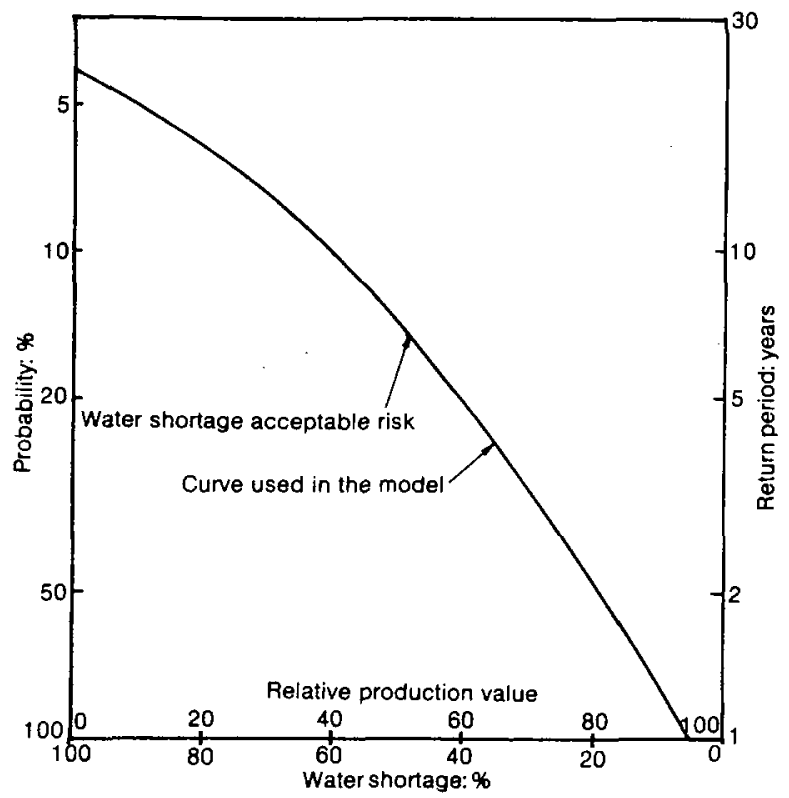

Fig. 7. Water shortage and crop fallure frequency analysis for vines 
maximum benefits. How was this problem dealt with in the preparation of the Authors model?

58. The final point in the Paper $(\$ 50)$ also caused some difficulty. However, in Chile it has been concluded that it is not necessary to rerun the model with different starting points in time if one works with probable rather than actual benefits in a given year. Thus the probability of obtaining the benefits of $B_{1}$ is calculated as $\left(B_{1} * n_{1}\right) /(N+1)$. where $n_{1}$ is the number of years in which $B_{1}$ is achieved and $N$ is the total number of years. It is possible in this way to calculate the probability of achieving any given level of benefit or alternatively, by summing each $(B * n) /(N+1)$, to calculate the most probable benefits associated with a given project over the whole hydrological cycle. This rather lengthy process is not necessary in fact as it can be shown that

$$
\sum \frac{B * n}{N+1}=\frac{\sum_{i=1}^{n} B_{i}}{N+1}
$$

This value is the expected value in every year and has been used as a constant annual flow in determining the net present value of the benefits at a given discount rate.

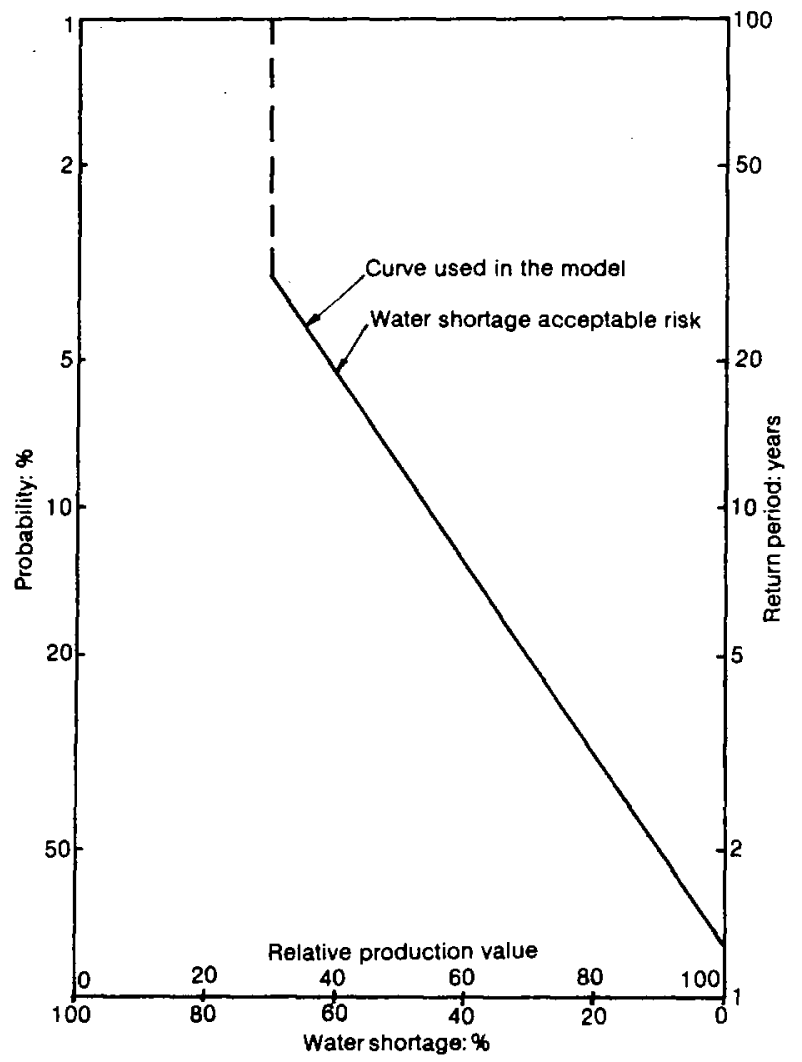

Fig. 8. Water shortage and crop failure frequency analysis for citrus fruits and deciduous trees 


\section{Dr White and Dr Christodoulou}

The reduction of the annual production of the various crops is calculated within the model by using empirical data prepared at the Water Development Department in Cyprus and based on experimental results in Cyprus and other countries (Figs 6 and 7 ). Fig. 8 shows the case for citrus fruits. With $40^{\circ} \%$ water shortage, which corresponds to a production value of $60 \%$ of the normal, the water shortage acceptable risk can be based on a 7.5 year return period. The acceptable probability of such a water shortage and crop failure is $14.8 \%$.

60. When water deficits occur an order of priorities is given as input to the model. For the Cyprus model the following order was
(a) domestic water supply
(b) citrus fruits
(c) bananas
(d) vines
(e) vegetables

When a deficit occurs in a particular month the demand with the first priority is satisfied first, then if possible the demand with the next priority is satisfied, and so on.

61. The problem of hydrological and economic security is dealt with in the model by using a set of empirical curves prepared at the Water Development Department in Cyprus and based on economical considerations and the estimated farmers' acceptance of crop failure risks within a probability of occurrences. These curves are also shown in Figs 6 and 7 and Fig. 8 shows how these curves are used for citrus fruits.

62. Consideration is being given to Dr Jarrold's suggested probabilistic way of obtaining benefits $(\$ 58)$ and what effect this will have on the timing of the model run and on the results. 